\section{Serial Sectioning via Microtomy (or, How To Get Over 100 Consecutive Serial Sections On One TEM Gird)}

\author{
David Elliott \\ University of Arizona College of Medicine \\ Tucson, $A Z$ \\ Elliott@arizona.edu
}

Serial sectioning of TEM blocks is a common technique. The more sections that are desired, the more complex the procedure becomes. Some of the problems faced are staining without losing or obscuring sections, appropriate block trimming, finding the area of interest in the next section, transferring sections to the grid. Here, I relate a set of techniques that permit the microtomist to regularly get over 100 consecutive serial sections on a single slot grid. Much of what is discussed below is not new, but by putting all of these techniques together I believe that a powerful tool for TEM is presented.

The first issue that must be addressed is how to stain the sections on the grid without losing sections or obscuring sections with staining artifacts (e.g. lead pepper). The simplest solution is to stain the sample with both uranium and lead prior to embedding and not stain the sections on the grid. Figure 1 shows an example of the contrast obtained using this technique. I Fix my samples and treat with osmium tetroxide as usual. Wash $3 \times 5 \mathrm{~min}$ in ddH2O. Incubate $1 \mathrm{~h}$ at RT with $2 \%$ Uranyl Acetate solution. Wash $3 \times 5 \mathrm{~min}$ in ddH2O. Dehydrate samples for $5 \mathrm{~min}$ through the following series $50 \%, 70 \%, 90 \%, 3 \times 100 \% \mathrm{EtOH}, 2 \times 1: 1 \mathrm{EtOH}$ :acetone. Incubate $1 \mathrm{~h}$ with a saturated solution of lead acetate in 1:1 EtOH:acetone. Wash samples with $2 \times 5$ min of 1:1 EtOH:acetone. Wash samples $2 \times 5$ min in acetone. Replace acetone with resin, change and cure as appropriate. I use Araldite/Embed-812 [1] and have found it to work well (14.5g Embed 812, 10.5g NMA, 5.0g DDS, and 0.54g DMP-30 cured overnight at $\left.55^{\circ} \mathrm{C}\right)$. Blocks must be appropriately hard to cut well using this technique.

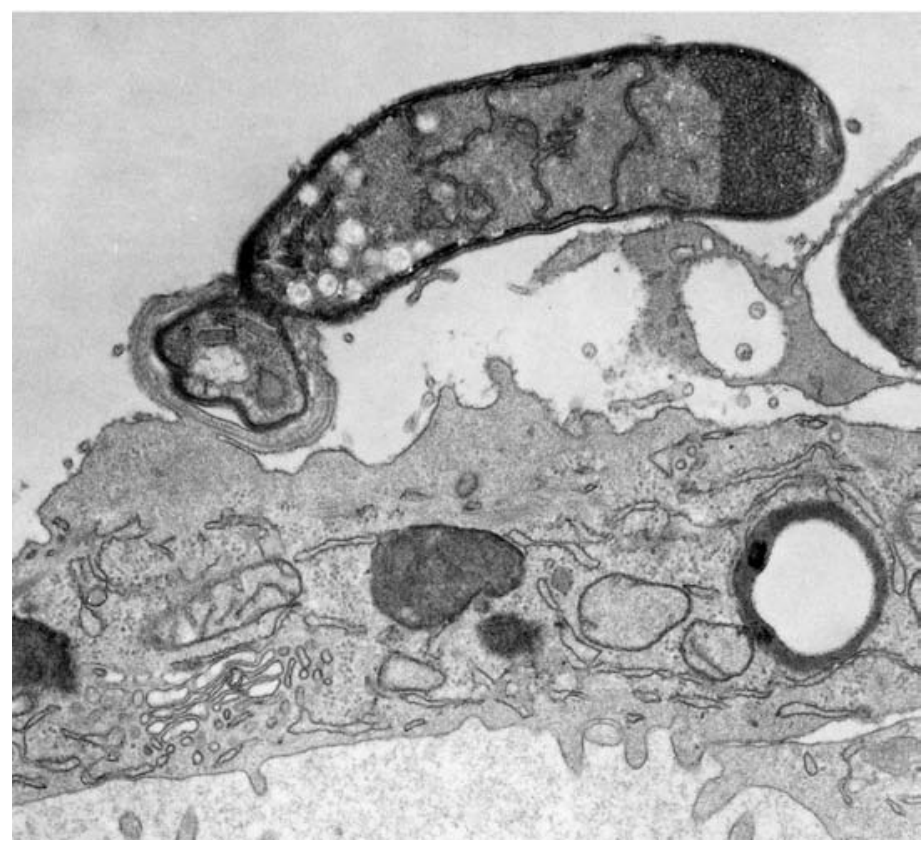

Figure 1 - This is a TEM of Cryptosporidium parvum invading a CacoII cell. The sample was embedded using this protocol. The sections were not stained on the grid.

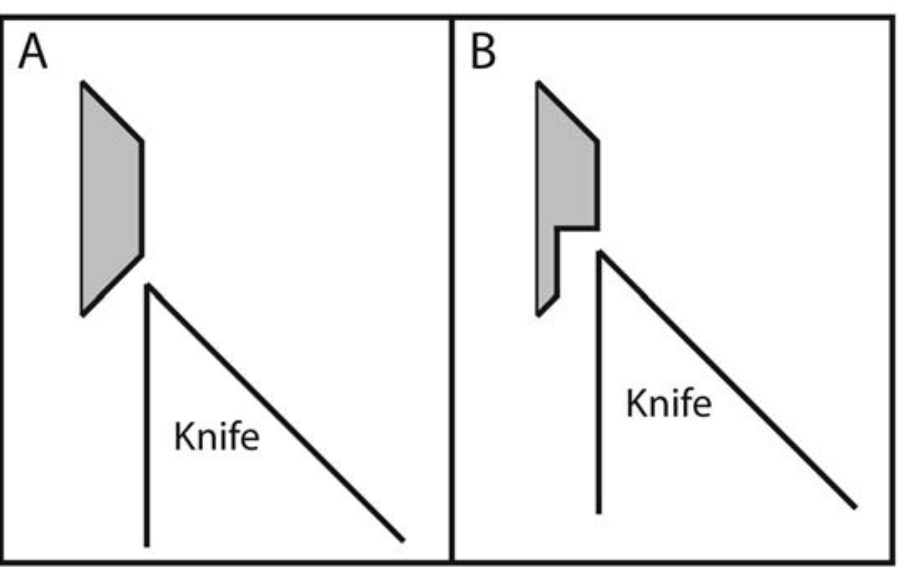

Figure 2 - This is a cartoon of the block face looking from the side. Panel A shows the normal block face shape. Panel B shows a block face that will cause each section to get higher by half as much as normal.

Once an appropriate block has been generated, the next issue is trimming the block face. Three things must be addressed: 1) section creep (each section is larger than the preceding section), 2) maximizing the number of sections that will fit on a grid (minimizing the height of the block face), and 3) making the top and bottom edge of the block face exactly parallel. Section creep is a function of the shape of the block face. If one looks at the side of a typical block face, both the top and bottom are sloped at a $45^{\circ}$ angle (figure 2A). This means that for each $100 \mathrm{~nm}$ cut off the block, the face height increases by $200 \mathrm{~nm}$. If one is only cutting a few sections, this increase is of no consequence, but if one is trying to maximize the number of sections on the grid, this is a serious concern. If the block face is cut as in figure $2 \mathrm{~B}$, then the creep is cut in half. The resin formula, above, is hard enough that this block face does not compress significantly while cutting. To maximize the number of sections on a grid the height of the block face must be minimized. Obviously, the block face can't be smaller than the object that you wish to view. I work with small cells and cut my block face about $10 \mu \mathrm{m}$ high. When hand trimming the block face, there is always an Corner to use for trimming error in how accurately one can cut the top and bottom of the block face. A $1^{\circ}$ departure from parallelism is normally an inconsequential error, but if one cuts 100 sections this error becomes a ribbon of sections that curves through $100^{\circ}$ of arc.

To make possible both a small block face with a parallel top and bottom, I have developed the following protocol. First, I trim the block face width. If using a $1 \times 2 \mathrm{~mm}$ slot grid, I recommend a $0.75-\mathrm{mm}$ wide face. Trim this dimension as you normally would. Next, a glass knife is mounted. Figure 3 shows a glass knife and which corner of the knife to use (If your knife has a good right corner, then reverse all of the following instructions). The first step is to rotate the

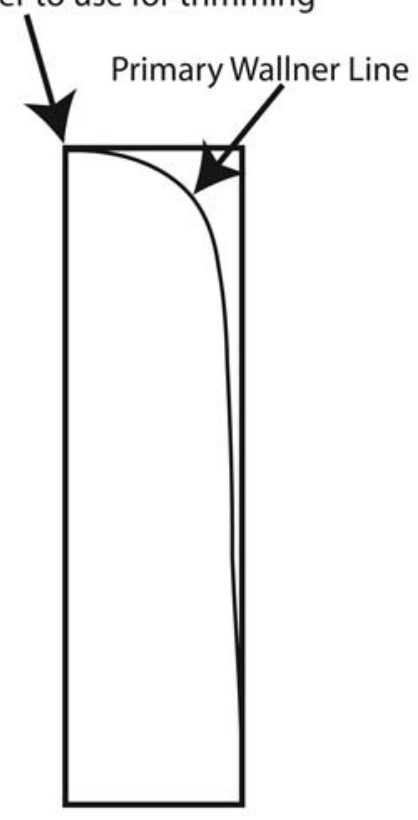

Figure 3 - This figure shows the correct corner of the glass knife to use for this protocol. This corner is sufficiently robust to withstand the use of this protocol. 


\section{Soft Imaging System -}

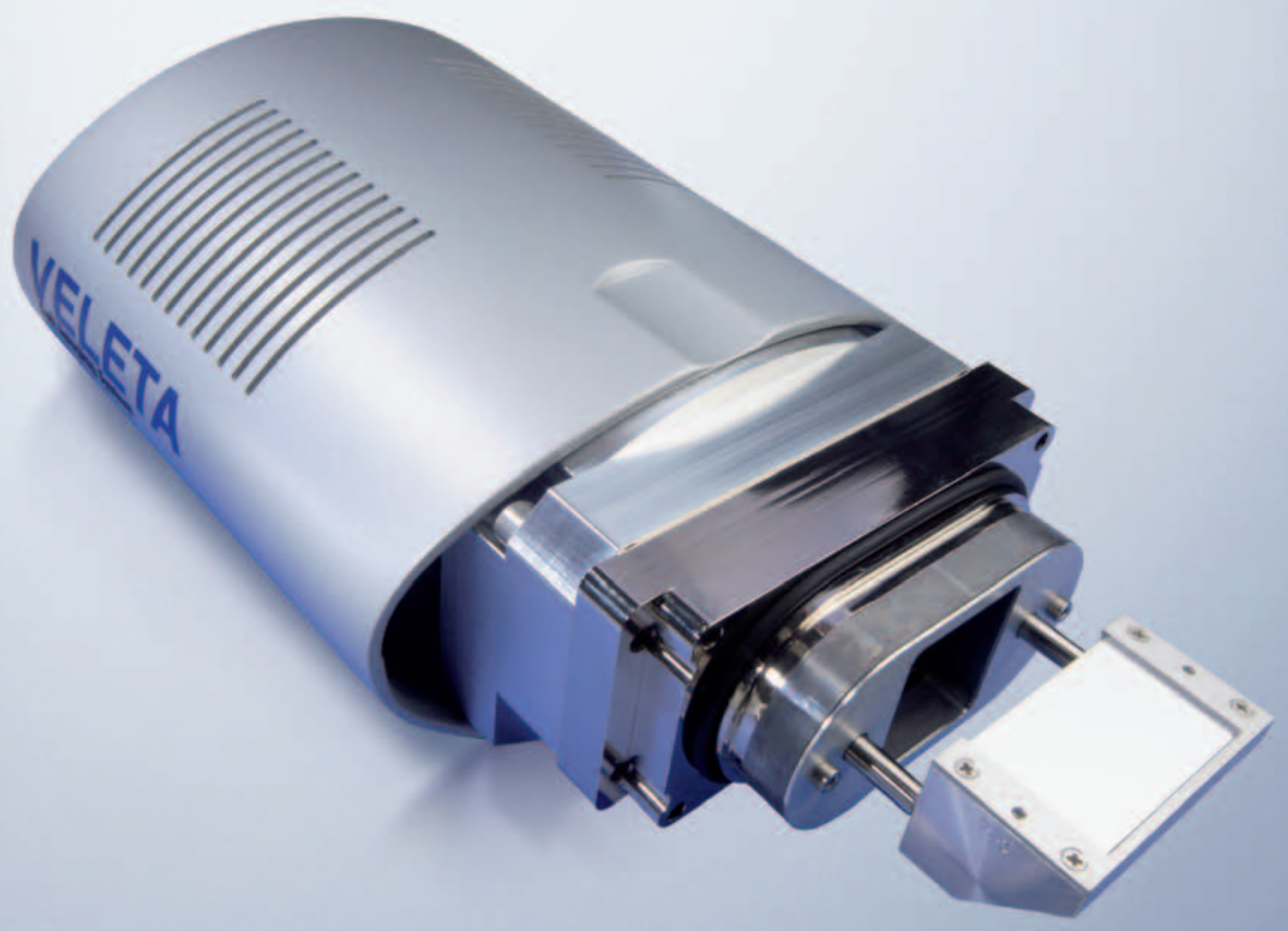

YOUR SPECIFICATIONS - OUR SOLUTION

VELETA - 2k x 2k SIDE-MOUNTED TEM CAMERA SOLUTION

Meeting the demands on today's TEM camera market - our new side-mounted TEM camera has the capabilities that customers are looking for in a $2 \mathrm{k} \times 2 \mathrm{k}$ camera. The new Veleta fills this description perfectly. If you're familiar with our MegaView III and Morada cameras, the Veleta's capabilities position it right between these two.

This new side-mounted TEM camera is our answer to the most current expectations on the TEM market. The Veleta is a Peltier-cooled camera featuring up to 2048 x 2048 highly sensitive pixels and has a 14-bit dynamic range. Veleta offers more than $10 \mathrm{fps}$ at full view and about $20 \mathrm{fps}$ at binning mode 2 .

Veleta is optimized for today's latest applications - such as tomography or immunogold labeling. Naturally the camera is applicable for all standard TEM imaging tasks.

TEM - Cameras |||||||||||||||

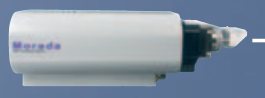

For detailed information please contact:

Olympus Soft Imaging Solutions

info.osis@olympus-sis.com

www.soft-imaging.net 


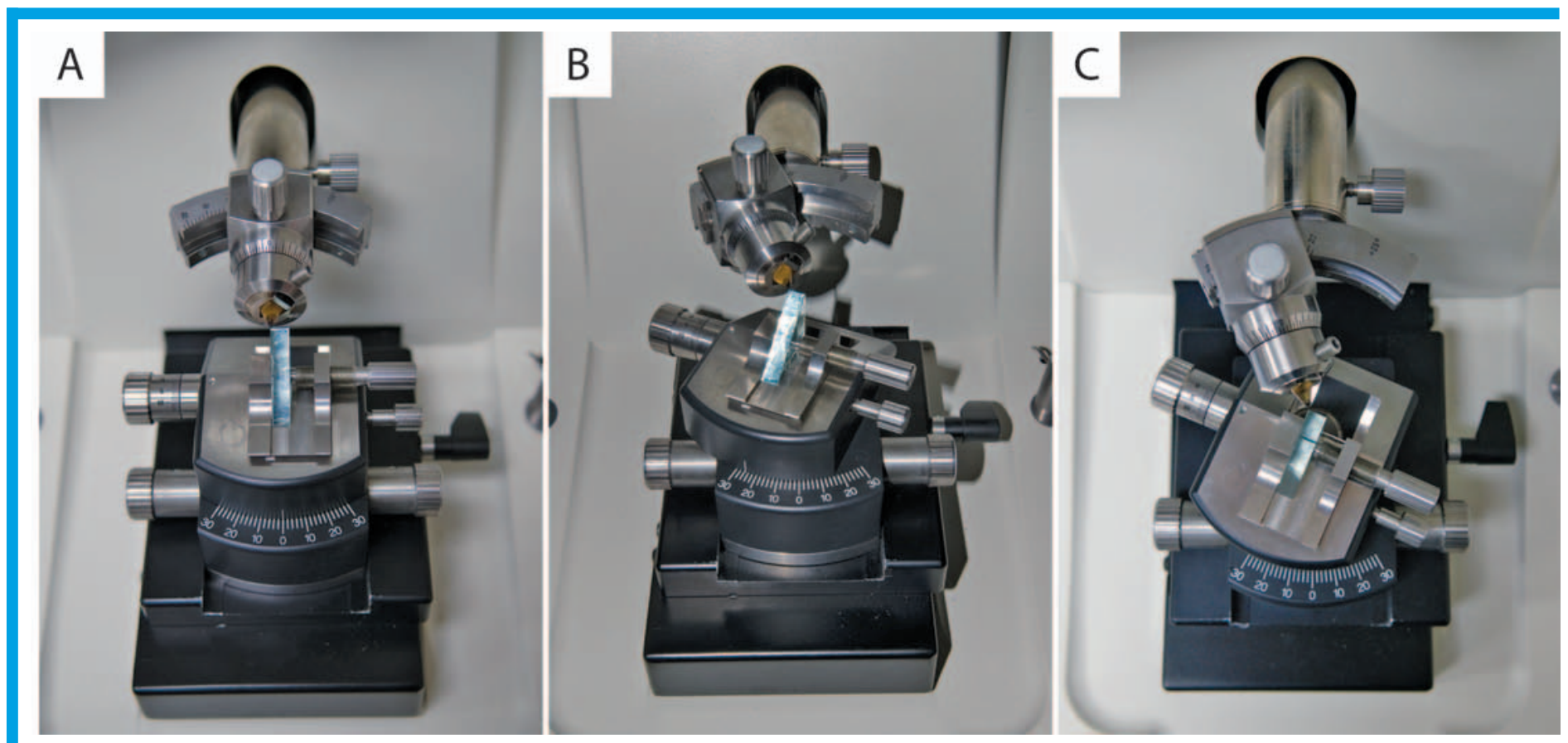

Figure 4 - This protocol involves some atypical adjustments of the block holder and the knife holder. Panel A shows the turning of the block holder needed for the first cut (figure 5B). Panel B \& $C$ show the block holder and the knife holder turned to the appropriate positions to permit the second cut (figure 5D). It is imperative that the block and block holder are not rotated between the first and second cut as this will cause the top and bottom of the block face to not be parallel.

entire block holder $90^{\circ}$ counter-clockwise (see figure 4A). Align the block and the knife as in figure $5 \mathrm{~A}$ and face the block until it is smooth. For all of these steps I recommend cutting $1 \mu \mathrm{m}$ thick sections. Next, move the knife corner to the right side of your sample (figure $5 \mathrm{~B}$ ) and cut into the block about $50 \mu \mathrm{m}$ ( 50 sections). This will leave a notch in the block seen in figure $5 \mathrm{C}$. Next angle the block $20^{\circ}$ counter-clockwise (see figure $4 \mathrm{~B} \& 4 \mathrm{C}$ ) using the adjustment knob that you would normally use to adjust the block face so that it is parallel to the knife-edge. Next, rotate the knife $25^{\circ}$ clockwise (see figure 4B \& 4C) and align the knife-corner and block face as seen in figure 5D. Begin sectioning into the block face to create a $45^{\circ}$ cut as seen in figure 5E. Continue sectioning until the face of the block is as thin as you want it. Figuring out where to align the knife at this step and determining how far to cut into the block requires some trial and error as one gains experience. If you start too far to the left then you will have to cut very far into the block until your face is as small as you want it. If you start cutting too far to the right then the block face will be thin before you have cut away sufficient waste. I find that a $10 \mu \mathrm{m}$ face is just visible, to my eyes, as a flat face. When done, the block face will look like figure 5E. Next, I set the knife angle and block angle back to $0^{\circ}$ (as shown in figure $4 \mathrm{~A}$ ), rotate the block $180^{\circ}$ and align the knife as shown in figure $5 \mathrm{~F}$. I then cut away the waste until the block looks like figure $5 \mathrm{G}$. At this point the block face is ready for sectioning. Rotate the block holder so that it is back in its normal position and, if necessary, rotate the block so that it is aligned as in figure 2B. At this point, the block face is about $0.75 \mathrm{~mm}$ wide by $10 \mu \mathrm{m}$ high.

Depending on the homogeneity of your sample and of the block, you may be ready to cut sections. However, if your sections do not form a nice, coherent ribbon, then you will need to use block glue. Many block glues are in the literature, but many of them are quite thick. I have found that many significantly increase the thickness of the sections (again, this is not a problem if one is cutting only a few sections, but....). The block glue I recommend is to dissolve/suspend Tackiwax $^{\text {tw }}$ [2] in chloroform. This suspension will be thick and gooey. Glob some on your block face and let the chloroform evaporate. Next, heat the Tackiwax with a hot pen [3] until the wax just melts (do not overheat your block or it will deform) and wick off excess wax with a piece of filter paper. In my experience, it is not possible for the coating of wax on the block to be too thin. This glue works very well to keep ribbons together. If your sections kink (this is normally because the hardness of your block is heterogeneous) it is possible to make the block face narrower. If it is also possible to cut out the part of the block that kinks, this will make things easier.

I cut a ribbon of section about $1.75 \mathrm{~mm}$ long. Depending on the size of the block face, this is about $80-120$ sections.

The last step is to transfer the sections to the slot grid without disturbing the ribbon. Put three pieces of double-sided tape one on top of the other on a petri-dish lid. Lightly stick just the edge of a slot grid (I use carbon coated formvar) to the tape so that the grid is suspended above the petri dish (the thickness of the tape will be the height of the grid above the dish). If the slot grid is too tightly attached to the tape then the formvar may rip when the grid is removed. Next,

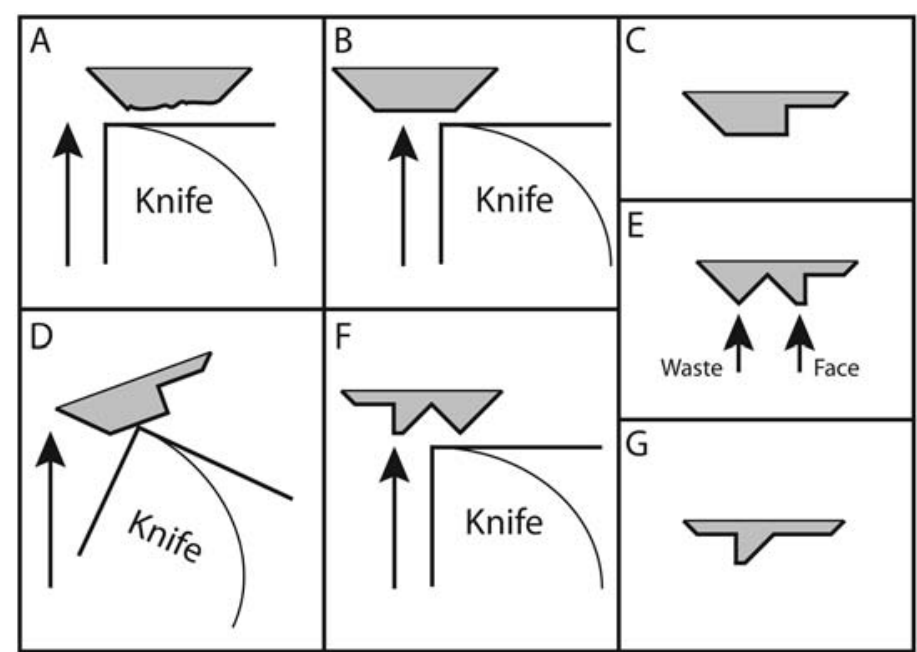

Figure 5 - These 7 panels show the alignment of the block and the knife for each step of the block trimming. Panels $A, B, D$, \& $F$ show the alignment of the cuts. Panels $C, E$, \& $G$ show the resultant shape of the block face as one looks down on it. 

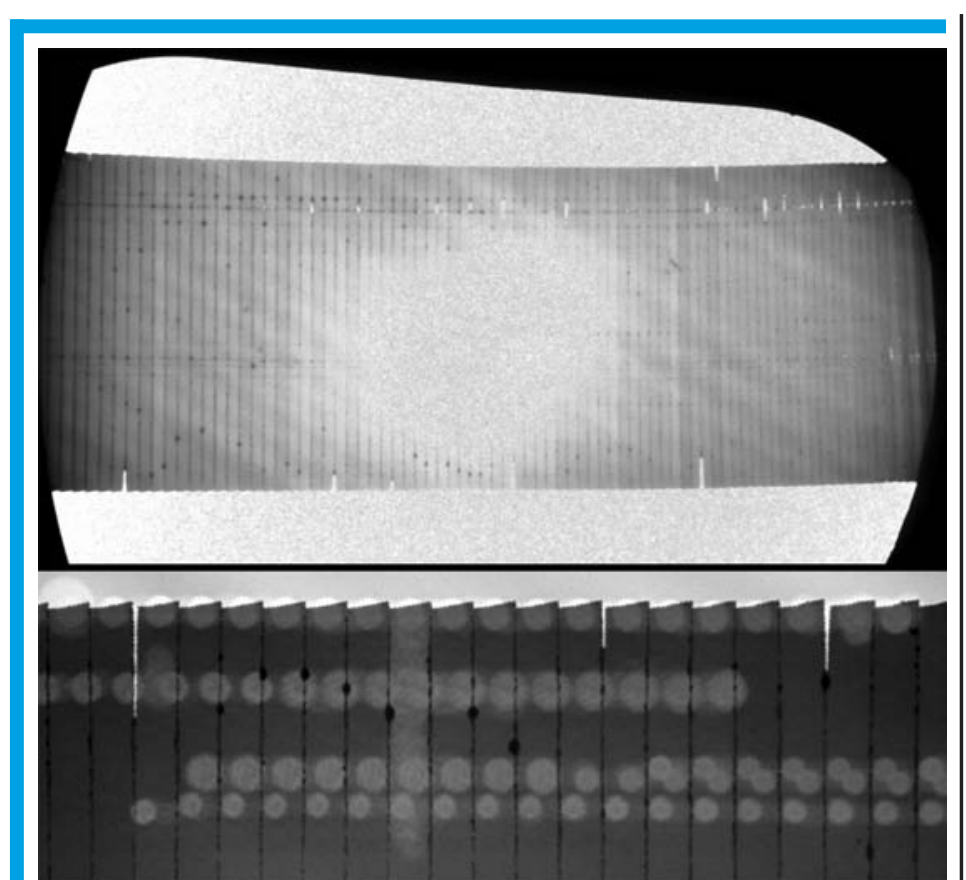

Figure 6 - Here is a ribbon showing 85 of the 102 sections on this grid. The lower panel shows the result of taking pictures on such a ribbon. It is easy to see that with the sections this small and perfectly aligned that it is simple to find your sample in the next section.

I use a second grid to pick up the ribbon. I use a clean slot grid with no formvar, shiny side up under the premise that the rough surface will not stick to the formvar grid as much as the shiny/smooth side would. I hold the grid with tweezers and move the ribbon to the center of the boat and align the ribbon with the open slot of your grid. I lower the grid onto the surface of the water until the ribbon is floating in the hole of the grid. Pick up the grid, with some water and the sections, and put it down on top of the formvar grid that I had previously stuck to the tape. This grid will be floating on the drop of water. I do the next step with a dissecting microscope. Align the slots of the two grids and wick out the water from between the two grids with a piece of filter paper. As the water is removed, the sections will settle down on the formvar of the lower slot grid. Let the grids sit until all water has evaporated (if there is water left between the grids when you try to remove the upper grid, it is very likely that you will rip the formvar). Next, remove the upper grid. At this point, you should have a grid ready for viewing in your TEM. An example of what the ribbon should look like can be seen in the upper panel of figure 6. One advantage of this technique is that the sections are in perfect register and the block face is small. This makes it very easy to find your sample in the next section. Figure 6 lower panel shows a part of a grid after viewing. The lighter circles are locations that I have taken pictures. You can see from the pattern where the samples were and how easy it is to find the next one.

\section{Acknowledgements}

I would like to thank Carol Cooke, Mike Delannoy, Carolyn Marks, and Marc Pypaert all of whom helped develop, or taught me, parts of this protocol.

\section{Endnotes}

Araldite/Embed-812 kit, Cat \# 13940, Electron Microscopy Science, 321 Morris Rd. Box 251, Fort Washington, PA 19034

2 Tackiwax, Cat \# 11444000, Boekel Scientific, 855 Pennsylvania Blvd., Feasterville, PA 19053

3 Hot Pen, Cat \# 72678, Electron Microscopy Science, 321 Morris Rd. Box 251, Fort Washington, PA 19034

\section{The Leaders in Lift Out Technology}

\author{
Now selling $5^{\text {th }}$ Generation \\ Ex-Situ Systems
}

INTRODUCING THE WORLDS FIRST AUTOMATED EX-SITU LIFT OUT SYSTEM

\section{"THE AUTO LIFT"}

The new "Auto Lift" is unmatched in maximizing TEM Sample throughput. No other instrument or technique is faster when working with a FIB. Great for Clean Room Environments!

\section{The Ex-Situ Method by Micro Optics of Florida, Inc.}

- The "Original" Lift Out Technique

- The New "Auto Lift" for high throughput.(Easy Upgrade for Existing Customers)

- Allows your FIB to do what it does best: Make TEM Samples

- Proven, Fast, Easy Technology Over 100 sold worldwide

- Success Rate is $95 \%$

- Cost Effective Systems including: The New "Auto Lift", Standard One and Two Manipulator systems, and a University model

Be sure to specify our Lift Out with your FIB!

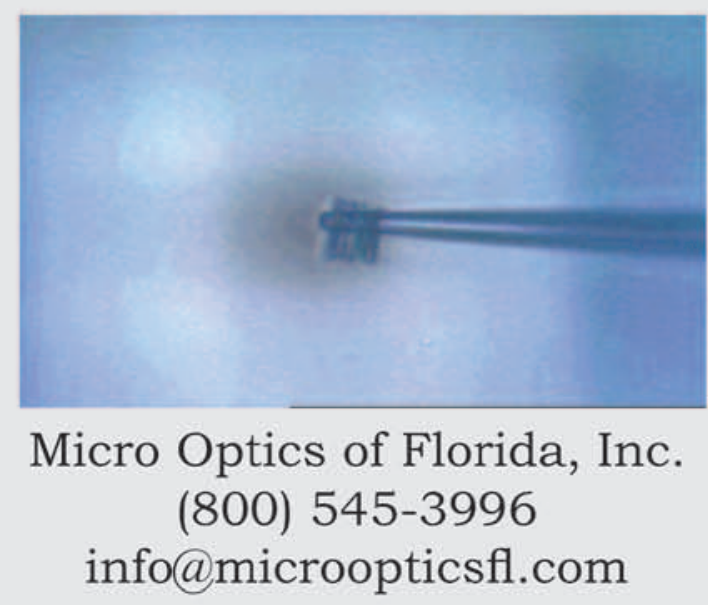

